\title{
The Illumination Bodies of a Simplex
}

\author{
E. Werner \\ Department of Mathematics, Case Western Reserve University, \\ Cleveland, $\mathrm{OH} 44106$, USA \\ emw2@po.cwru.edu \\ and \\ Université de Lille 1, Ufr de Mathematique, \\ 59655 Villeneuve d' Ascq, France
}

Let $P$ be a polytope in $\mathbf{R}^{n}$ and define $P(\delta)$, the $\delta$-parallel body of $P$, by

$$
P(\delta)=\left\{x \in \mathbf{R}^{n}: \operatorname{dist}(x, P) \leq \delta\right\} .
$$

It is a classical result that the volume of $P(\delta)$ is an $n$th degree polynomial in $\delta$ whose coefficients (when properly normalized) are the elementary mixed volumes of the polytope $P$.

At the beginning of this century Blaschke and his school of differential geometry began the investigation of affine parallel bodies. These so called $\delta$-floating bodies arose in connection with notions of affine differential geometry, for example, affine surface area. This affine surface area was originally only defined for convex bodies in $\mathbf{R}^{n}$ with a sufficiently smooth boundary. However, it was desired to extend this definition to arbitrary convex bodies as affine surface area occurs naturally in important questions, for instance, in the approximation of convex bodies by polytopes (see the survey of Gruber [G]).

Various such extensions have been given within the last few years, for example, by Leichtweiss [Le], Lutwak [Lu], Schütt and Werner [SW], and Werner [W]. In most of these extensions one considers volume differences

$$
\operatorname{vol}_{n}(K)-\operatorname{vol}_{n}\left(K_{\delta}\right) \quad\left(\text { resp. } \operatorname{vol}_{n}\left(K^{\delta}\right)-\operatorname{vol}_{n}(K)\right),
$$

where $K_{\delta}$ (resp. $K^{\delta}$ ) are convex bodies related to $K$, namely the floating body (see [Le] or [SW] for the definition) (resp. the illumination body). It is known that, for sufficiently smooth bodies, the above volume differences behave like $\delta^{2 /(n+1)}$ (see [Le], [SW], and [W]). 
In [S] Schütt studied such volume differences for polytopes $P$ in $\mathbf{R}^{n}$ and their floating bodies. He obtained - among other things-the following result:

$$
\lim _{\delta \rightarrow 0} \frac{\operatorname{vol}_{n}(P)-\operatorname{vol}_{n}\left(P_{\delta}\right)}{\delta \ln (1 / \delta)}=\# \frac{\{\text { flags of } P\}}{n ! n^{n}},
$$

where a flag is an $n$-tuple $\left(f_{0}, f_{1}, \ldots, f_{n-1}\right)$ such that each $f_{i}$ is an $i$-dimensional face of $P$ and such that $f_{i} \subset f_{i+1}$ for all $i, 0 \leq i \leq n-2$. Here and elsewhere throughout the paper the symbol \# stands for the number of objects under consideration.

In this paper we study volume differences for polytopes and their illumination bodies. It is known that this volume difference is also a polynomial in $\delta$ of degree $n$ (see below) but-in contrast to the classical $\delta$-parallel body - not much is known about the coefficents of this polynomial. We give those in the case of a simplex in $\mathbf{R}^{n}$.

For a convex body $K$ in $\mathbf{R}^{n}$ and $\delta>0$, the illumination body $K^{\delta}$ is the convex body

$$
K^{\delta}=\left\{x \in \mathbf{R}^{n}: \operatorname{vol}_{n}(\operatorname{co}[x, K])-\operatorname{vol}_{n}(K) \leq \delta\right\},
$$

where $c o[x, K]$ denotes the convex hull of $x$ and $K$.

We first mention some known results. For completeness we include outlines of the proofs.

Theorem. For every polytope $P \subseteq \mathbf{R}^{n}, \operatorname{vol}_{n}\left(P^{\delta}\right)-v^{\prime} l_{n}(P)$ is a polynomial in $\delta$ of degree $n$.

Proposition. For every polytope $P \subseteq \mathbf{R}^{n}$,

$$
\lim _{\delta \rightarrow 0} \frac{v o l_{n}\left(P^{\delta}\right)-v o l_{n}(P)}{\delta}=n \#\{(n-1) \text {-dimensional faces of } P\} .
$$

Proposition. For a cube $C$ in $\mathbf{R}^{n}$, we have

$$
\operatorname{vol}_{n}\left(C^{\delta}\right)-\operatorname{vol}_{n}(C)=\sum_{k=1}^{n}\left(\begin{array}{l}
n \\
k
\end{array}\right) \frac{(2 n)^{k}}{k !} \frac{\delta^{k}}{\left(\operatorname{vol}_{n}(C)\right)^{k-1}}
$$

Outline of the Proofs. Let $P$ be a polytope in $\mathbf{R}^{n}$ and let $\delta>0, \delta$ small, be given. We first give a description of $P^{\delta}$. To do so associate, for all $m, 1 \leq m \leq n$, the "visibility cone" $C_{n-m}$ to each $(n-m)$-dimensional face $F_{n-m}$ of $P . F_{n-m}$ is the intersection of a certain number of adjacent $(n-1)$-dimensional faces. Then $C_{n-m}$ is the intersection of all those half-spaces determined by the adjacent $(n-1)$-dimensional faces of $F_{n-m}$ that do not contain $P$. Hence $C_{n-m}$ is the cone from where the face $F_{n-m}$ and all $(n-1)$-dimensional faces adjacent to $F_{n-m}$ can be seen.

We now give an inductive procedure of how to obtain $P^{\delta}$. "Above" an $(n-1)$ dimensional face $F_{n-1}$ the boundary of $P^{\delta}$ consists of a hyperplane parallel to this face at distance $n \delta /$ vol $_{n-1}\left(F_{n-1}\right)$. Consequently, for $\delta$ small enough, the contribution of each $(n-1)$-dimensional face to $v_{o l} l_{n}\left(P^{\delta}\right)-v o l_{n}(P)$ is $\delta$.

Thus we have shown the first proposition above if we show that the other $(n-m)$ dimensional faces, $m>1$, contribute only higher-order terms in $\delta$. Consider next, 
therefore, an $(n-2)$-dimensional face $F_{n-2}$ with its visibility cone $C_{n-2} . F_{n-2}$ is contained in two $(n-1)$-dimensional faces $F_{n-1}^{i}, i=1,2$. The boundary of $P^{\delta}$ above these $(n-1)$-dimensional faces is already determined by the hyperplanes $H^{i}$ parallel to $F_{n-1}^{i}$ at distance $h^{i}=n \delta /$ vol $_{n-1}\left(F_{n-1}^{i}\right), i=1,2$. Then the part of $P^{\delta}$ in $C_{n-2}$ is obtained as follows. The intersection of the $H^{i}$ 's with the prolongated $(n-1)$-dimensional faces $F_{n-1}^{i}, i=1,2$, is taken and then the convex hull of this set with $F_{n-2}$ is also taken. Thus the contribution of an $(n-2)$-dimensional face to $v_{n} l_{n} P^{\delta}-v o l_{n} P$ is proportional to $h_{1} \cdot h_{2}$, hence to $\delta^{2}$, modulo a factor that depends, e.g., on the volumes of the involved faces and on the angle of intersection of $F_{n-1}^{1}$ and $F_{n-1}^{2}$ at $F_{n-2}$.

We proceed in this way to obtain the statement of the theorem.

In the case of a cube $C$ it is easy to compute the exact coefficients of the powers of $\delta$, as the angles of intersection of all the respective faces are $\pi / 2$. Therefore the contribution of an $(n-m)$-dimensional face to $\operatorname{vol}_{n}\left(C^{\delta}\right)-\operatorname{vol}_{n}(C)$ is $(1 / m !)\left(\left(n^{m} \cdot \delta^{m}\right) /\left(\operatorname{vol}_{n}(C)\right)^{m-1}\right)$ and there are $\left(\begin{array}{c}n \\ n-m\end{array}\right) 2^{m}$ faces of dimension $(n-m)$.

We now consider the case of a simplex $S_{n}$ in $\mathbf{R}^{n}$. As $\operatorname{vol}_{n}\left(S_{n}^{\delta}\right)-\operatorname{vol}_{n}\left(S_{n}\right)$ is invariant under affine transformations $T$ with $\operatorname{det} T=1$, it is sufficient to consider the case of a regular simplex, i.e., a simplex with congruent $(n-1)$-dimensional faces. We define for all $i, 1 \leq i \leq n$, the numbers $L_{i} \in \mathbf{R}$ and the points $l_{i} \in \mathbf{R}^{n}$ recursively as follows:

$$
\begin{gathered}
L_{1}=1, \quad L_{i}=\left(1-\sum_{j=1}^{i-1}\left(\frac{L_{j}}{j+1}\right)^{2}\right)^{1 / 2}, \\
l_{1}=\left(L_{1}, 0, \ldots, 0\right), \quad l_{i}=\left(\frac{L_{1}}{2}, \frac{L_{2}}{3}, \ldots, \frac{L_{i-1}}{i}, L_{i}, 0 \cdots 0\right) .
\end{gathered}
$$

We also write $l_{0}=(0, \ldots, 0)$ for the 0 -vector.

Notice that for all $i, i \geq 1,\left\|l_{i}\right\|_{2}=1$ and $\left\|l_{i+1}-l_{i}\right\|_{2}=1$, where $\|\cdot\|_{2}$ is the usual Euclidean norm in $\mathbf{R}^{n}$. If $s>0$, then the convex hull $c o\left\{s l_{i}: 0 \leq i \leq n\right\}$ of the points $s l_{i}, 0 \leq i \leq n$, is a regular simplex $S_{n}$ in $\mathbf{R}^{n}$ with sidelength $s$ and we have

$$
\operatorname{vol}_{n}\left(S_{n}\right)=\frac{s^{n}}{n !} \prod_{i=1}^{n} L_{i}
$$

Theorem 1. Let $S_{n}$ be a simplex in $\mathbf{R}^{n}$. Then we have, for all $\delta \geq 0$,

$$
\operatorname{vol}_{n}\left(S_{n}^{\delta}\right)-\operatorname{vol}_{n}\left(S_{n}\right)=\sum_{k=1}^{n}\left(\begin{array}{l}
n \\
k
\end{array}\right)\left(\begin{array}{c}
n+k \\
n
\end{array}\right) \frac{\delta^{k}}{\left(\operatorname{vol}_{n}\left(S_{n}\right)\right)^{k-1}} .
$$

To prove Theorem 1 we need to describe $S_{n}^{\delta}$. As already mentioned it is sufficient to consider a regular simplex.

Proposition 2. Let $S_{n}$ be a regular simplex in $\mathbf{R}^{n}$ with sidelength s. Let $\delta>0$ be given and let $h=n \delta /$ vol $_{n-1}\left(S_{n-1}\right)$ where $S_{n-1}$ is an $(n-1)$-dimensional face of $S_{n}$. Then

$$
S_{n}^{\delta}=c o\left\{s l_{k}+\frac{h}{L_{n}}\left(l_{k}-l_{j}\right): 0 \leq k \leq n, 0 \leq j \leq n, j \neq k\right\} \text {. }
$$




\section{Proof. Define $M$ by}

$$
M=c o\left\{s l_{k}+\frac{h}{L_{n}}\left(l_{k}-l_{j}\right): 0 \leq k \leq n, 0 \leq j \leq n, j \neq k\right\} .
$$

For all $m, 1 \leq m \leq n$, we associate the "visibility cone" $C_{n-m}$ to each $(n-m)$ dimensional face $S_{n-m}$ of $S_{n}$. Observe that because of symmetry it is enough to consider the faces

$$
S_{n-m}=\operatorname{co}\left\{s l_{k}: 0 \leq k \leq n-m\right\} .
$$

$S_{n-m}$ is the intersection of its $m$ adjacent $(n-1)$-dimensional faces $S_{n-1}^{i}, 0 \leq i \leq m-1$. We obtain $C_{n-m}$ by prolongating each of these adjacent $m$ faces and by taking $S_{n-m}$ as a base for the cone.

More precisely,

$C_{n-m}=\operatorname{co}\left[S_{n-m} \cup\left\{s l_{k}+t\left(l_{k}-l_{n-j}\right): 0 \leq k \leq n-m, 0 \leq j \leq m-1, t \in \mathbf{R}, t \geq 0\right\}\right]$.

$C_{n-m}$ is the cone from where the $m(n-1)$-dimensional faces $S_{n-1}^{i}$ adjacent to $S_{n-m}$ can be seen but no other $(n-1)$-dimensional face of $S_{n} . C_{n-1}$ is the cone from where just $S_{n-1}$ is seen. Notice also that taking $t=h / L_{n}$ fixed in the definition of $C_{n-m}, M$ is the union of $S_{n}$ and all the visibility cones $C_{n-m}, 1 \leq m \leq n$, with this fixed $t$.

We show first that $M \subset S_{n}^{\delta}$. To do so we show:

Lemma 3. Let $x \in M$ such that $x$ is in an $(n-1)$-dimensional face of $M$. Then

$$
\operatorname{vol}_{n}\left(\operatorname{co}\left[x, S_{n}\right]\right)-\operatorname{vol}_{n}\left(S_{n}\right)=\delta \text {. }
$$

Proof of the Lemma. Let $x \in M$ such that $x$ is in an (n-1)-dimensional face of $M$. Then $x \in C_{n-m}$ for some $m$. Consequently, $x$ is a convex combination of the points $s l_{k}+\left(h / L_{n}\right)\left(l_{k}-l_{n-j}\right), 0 \leq k \leq n-m, 0 \leq j \leq m-1$, i.e.,

$$
x=\sum_{j=0}^{m-1} \sum_{k=0}^{n-m} \lambda_{k}^{j}\left(s l_{k}+\frac{h}{L_{n}}\left(l_{k}-l_{n-j}\right)\right)
$$

with

$$
\sum_{j=0}^{m-1} \sum_{k=0}^{n-m} \lambda_{k}^{j}=1
$$

As $x \in C_{n-m}$,

$$
\operatorname{vol}_{n}\left(\operatorname{co}\left[x, S_{n}\right]\right)-\operatorname{vol}_{n}\left(S_{n}\right)=\frac{1}{n} \operatorname{vol}_{n-1}\left(S_{n-1}\right) \sum_{i=0}^{m-1} \operatorname{dist}\left(x, S_{n-1}^{i}\right),
$$

where $\operatorname{dist}\left(x, S_{n-1}^{i}\right)$ is the distance of $x$ to the $(n-1)$-dimensional face $S_{n-1}^{i} . S_{n-1}^{i}$ is the $i$ th $(n-1)$-dimensional face that can be seen from $x$, i.e., for $0 \leq i \leq m-1$,

$$
S_{n-1}^{i}=c o\left[\left\{s l_{k}: 0 \leq k \leq n-m\right\} \cup\left\{s l_{n-k}: 0 \leq k \leq m-1, k \neq i\right\}\right] .
$$


As $S_{n-1}^{0}$ sits in the hyperplane with the $x_{n}$-coordinate equal to 0 ,

$$
\operatorname{dist}\left(x, S_{n-1}^{0}\right)=\mid x_{n} \text {-coordinate of } x \mid=h \cdot \sum_{k=0}^{n-m} \lambda_{k}^{0}
$$

For $1 \leq i \leq m-1, m \geq 2$,

$$
\operatorname{dist}\left(x, S_{n-1}^{i}\right)=\frac{\left|\left\langle N^{i}, x\right\rangle\right|}{\left\|N^{i}\right\|_{2}}
$$

where $N^{i}=\left(y_{1}, y_{2}, \ldots, y_{n}\right)$ is a normal to $S_{n-1}^{i}$. As $0 \in S_{n-1}^{i}$, we can choose $N^{i}$ such that

$$
\begin{gathered}
y_{n-i}=\prod_{\substack{k=0 \\
k \neq 0}}^{i} L_{n-i+k}, \\
y_{n-i+1}=-\frac{\prod_{\substack{k=0 \\
k \neq 1}}^{i} L_{n-i+k}}{n-i+1}, \\
y_{n-i+2}=-\frac{\prod_{\substack{k=0 \\
k \neq 2}}^{i} L_{n-i+k}}{n-i+2},
\end{gathered}
$$

and so forth up to

$$
y_{n}=-\frac{\prod_{\substack{k=0 \\ k \neq i}}^{i} L_{n-i+k}}{n}
$$

and all the other coordinates of $N^{i}$ equal to 0 . Hence

$$
\begin{aligned}
\left\langle x, N^{i}\right\rangle= & -\prod_{\substack{k=0 \\
k \neq 0}}^{i} L_{n-i+k}\left(\sum_{j=0}^{i-1} \sum_{k=0}^{n-m} \lambda_{k}^{j} \frac{h}{L_{n}} \frac{L_{n-i}}{(n-i+1)}+\sum_{k=0}^{n-m} \lambda_{k}^{i} \frac{h}{L_{n}} L_{n-i}\right) \\
& +\prod_{\substack{k=0 \\
k \neq 1}}^{i} L_{n-i+k}\left(\sum_{j=0}^{i-2} \sum_{k=0}^{n-m} \lambda_{k}^{j} \frac{h}{L_{n}} \frac{L_{n-i+1}}{(n-i+2)}+\sum_{k=0}^{n-m} \lambda_{k}^{i-1} \frac{h}{L_{n}} L_{n-i+1}\right) \\
& +\cdots \\
& +\prod_{\substack{k=0 \\
k \neq i-1}}^{i} L_{n-i+k}\left(\sum_{k=0}^{n-m} \lambda_{k}^{0} \frac{h}{L_{n}} \frac{L_{n-1}}{n}+\sum_{k=0}^{n-m} \lambda_{k}^{1} \frac{h}{L_{n}} L_{n-1}\right) \\
& +\prod_{\substack{k=0 \\
k \neq i}}^{i} L_{n-i+k} \sum_{k=0}^{n-m} \lambda_{k}^{0} \frac{h}{L_{n}} L_{n}
\end{aligned}
$$




$$
\begin{aligned}
=h \cdot \prod_{k=0}^{i-1} L_{n-i+k}\left\{\sum _ { k = 0 } ^ { n - m } \lambda _ { k } ^ { 0 } \left[\frac{1}{n}+\frac{1}{n(n-1)}+\frac{1}{(n-1)(n-2)}+\cdots\right.\right. \\
\left.\quad+\frac{1}{(n-i+2)(n-i+1)}-\frac{1}{n-i+1}\right] \\
+\sum_{k=0}^{n-m} \lambda_{k}^{1}\left[\frac{1}{n-1}+\frac{1}{(n-1)(n-2)}+\cdots\right. \\
+\cdots \\
\quad-\sum_{k=0}^{n-m} \lambda_{k}^{i}
\end{aligned}
$$

Now observe that, for all $0 \leq k \leq i-2$,

$$
\frac{1}{n-k}+\frac{1}{(n-k)(n-k-1)}+\cdots+\frac{1}{(n-i+2)(n-i+1)}-\frac{1}{n-i+1}=0
$$

hence

$$
\left\langle x, N^{i}\right\rangle=-h \prod_{k=0}^{i-1} L_{n-i+k} \sum_{k=0}^{n-m} \lambda_{k}^{i}
$$

As, for all $j$,

$$
\prod_{\substack{k=0 \\ k \neq j}}^{i} L_{n-i+k}^{2}+\frac{\prod_{\substack{k=0 \\ k \neq(j+1)}}^{i} L_{n-i+k}^{2}}{(n-i+j+1)^{2}}=\prod_{\substack{k=0 \\ k \neq(j+1)}}^{i} L_{n-i+k}^{2},
$$

we get that $\left\|N^{i}\right\|_{2}=\prod_{k=0}^{i-1} L_{n-i+k}$, hence

$$
\operatorname{vol}_{n}\left(\operatorname{co}\left[x, S_{n}\right]\right)-\operatorname{vol}_{n}\left(S_{n}\right)=\frac{1}{n} \operatorname{vol}_{n-1}\left(S_{n-1}\right) h \cdot \sum_{i=0}^{m-1} \sum_{k=0}^{n-m} \lambda_{k}^{i}=\delta .
$$

This finishes the proof of Lemma 3, which shows that $M \subset S_{n}^{\delta}$.

We continue with the proof of Proposition 2 and for the converse suppose $S_{n}^{\delta} \not \subset M$. Then there is an $x \in S_{n}^{\delta}$ such that $x \notin M$. However, $x \in C_{n-m}$ for some $m$. As $x \notin M, x$ has a strictly positive distance to the $(n-1)$-dimensional face $F$ of $M$ which sits in $C_{n-m}$, i.e., the face which is spanned by the vectors $s l_{k}+\left(h / L_{n}\right)\left(l_{k}-l_{n-j}\right), 0 \leq k \leq n-m$, $0 \leq j \leq m-1$. This implies that

$$
\operatorname{co}\left[x, S_{n}\right] \supset \operatorname{co}\left[y, S_{n}\right]
$$


for a suitable $y \in F$ and consequently, by Lemma 3,

$$
\operatorname{vol}_{n}\left(\operatorname{co}\left[x, S_{n}\right]\right)-\operatorname{vol}_{n}\left(S_{n}\right)>\delta,
$$

a contradiction to the definition of $S_{n}^{\delta}$.

Lemma 4. Let $1 \leq m \leq n$. The contribution of an $(n-m)$-dimensional face to $\operatorname{vol}_{n}\left(S_{n}^{\delta}\right)-\operatorname{vol}_{n}\left(S_{n}\right)$ is

$$
\sum_{k=m}^{n}\left(\begin{array}{l}
n \\
k
\end{array}\right) \frac{\delta^{k}}{\left(\operatorname{vol}_{n}\left(S_{n}\right)\right)^{k-1}} \sum_{i=0}^{k-m}(-1)^{i}\left(\begin{array}{c}
k \\
m+i
\end{array}\right) .
$$

Proof. An $(n-m)$-dimensional face of $S_{n}$ is a simplex $S_{n-m}$ in $\mathbf{R}^{n-m}$. Because of symmetry we can assume that $S_{n-m}$ is the convex hull of the points $s l_{k}, 0 \leq k \leq n-m$. From the description of $S_{n}^{\delta}$ given in Proposition 2 we see that the contribution of the face $S_{n-m}$ to $\operatorname{vol}_{n}\left(S_{n}^{\delta}\right)-\operatorname{vol}_{n}\left(S_{n}\right)$ is the volume of the $n$-dimensional convex polytope $P^{n-m}$ that has $S_{n-m}$ as an $(n-m)$-dimensional face and, at each extreme point of $S_{n-m}$, "stick out the missing directions" $-l_{n},-l_{n-1}, \ldots,-l_{n-m+1}$ (resp. combinations of those). More precisely,

$$
\begin{aligned}
P^{n-m}=c o[ & \left\{s l_{k}: 0 \leq k \leq n-m\right\} \\
& \left.\cup\left\{s l_{k}+\frac{h}{L_{n}}\left(l_{k}-l_{n-i}\right): 0 \leq k \leq n-m, 0 \leq i \leq m-1\right\}\right] .
\end{aligned}
$$

To compute $\operatorname{vol}_{n}\left(P^{n-m}\right)$ we fix successively the $x_{n}-, x_{n-1}-, \ldots$ coordinates.

If $m=1$, we are already in the situation discussed below and nothing is to be done. Otherwise consider the $x_{n}$-coordinate. Observe that $-h \leq x_{n} \leq 0$. Fix the $x_{n}$-coordinate. Then an $(n-1)$-dimensional section $P_{n-1}^{n-m}$ of $P^{n-m}$ with a fixed $x_{n}$-coordinate is

$$
\begin{gathered}
P_{n-1}^{n-m}=c o\left[\left\{s l_{k}-\frac{x_{n}}{L_{n}}\left(l_{k}-l_{n}\right): 0 \leq k \leq n-m\right\}\right. \\
\cup\left\{s l_{k}+\frac{h}{L_{n}}\left(l_{k}-l_{n-i}\right)+\frac{x_{n}}{L_{n}}\left(l_{n}-l_{n-i}\right):\right. \\
0 \leq k \leq n-m, 1 \leq i \leq m-1\}] .
\end{gathered}
$$

$\operatorname{vol}_{n-1}\left(P_{n-1}^{n-m}\right)$ does not change if we shift $P_{n-1}^{n-m}$ by the fixed vector $\left(x_{n} / L_{n}\right) l_{n}$ and instead of $P_{n-1}^{n-m}$ we consider the shifted polytope $S_{n-1}^{n-m}$ with

$$
\begin{aligned}
& S_{n-1}^{n-m}=c o[\left\{l_{k}\left(s-\frac{x_{n}}{L_{n}}\right): 0 \leq k \leq n-m\right\} \\
& \cup\left\{l_{k}\left(s-\frac{x_{n}}{L_{n}}\right)+\left(\frac{h}{L_{n}}+\frac{x_{n}}{L_{n}}\right)\left(l_{k}-l_{n-i}\right):\right. \\
&0 \leq k \leq n-m, 1 \leq i \leq m-1\}] .
\end{aligned}
$$


If $m=2$, we are done. Otherwise we next consider the $x_{n-1}$-coordinate. Observe that

$$
-\left(\frac{h}{L_{n}}+\frac{x_{n}}{L_{n}}\right) L_{n-1} \leq x_{n-1} \leq 0 .
$$

We keep the $x_{n-1}$-coordinate fixed. Then an $(n-2)$-dimensional section $P_{n-2}^{n-m}$ of $S_{n-1}^{n-m}$ is

$$
\begin{gathered}
P_{n-2}^{n-m}=c o\left[\left\{l_{k}\left(s-\frac{x_{n}}{L_{n}}\right)-\frac{x_{n-1}}{L_{n-1}}\left(l_{k}-l_{n-1}\right): 0 \leq k \leq n-m\right\}\right. \\
\cup\left\{l_{k}\left(s-\frac{x_{n}}{L_{n}}\right)+\left(\frac{h}{L_{n}}+\frac{x_{n}}{L_{n}}\right)\left(l_{k}-l_{n-i}\right)+\frac{x_{n-1}}{L_{n-1}}\left(l_{n-1}-l_{n-i}\right):\right. \\
0 \leq k \leq n-m, 2 \leq i \leq m-1\}] .
\end{gathered}
$$

After shifting by the vector $\left(x_{n-1} / L_{n-1}\right) l_{n-1}$ we get

$$
\begin{gathered}
S_{n-2}^{n-m}=c o\left[\left\{l_{k}\left(s-\frac{x_{n}}{L_{n}}-\frac{x_{n-1}}{L_{n-1}}\right): 0 \leq k \leq n-m\right\}\right. \\
\cup\left\{l_{k}\left(s-\frac{x_{n}}{L_{n}}-\frac{x_{n-1}}{L_{n-1}}\right)+\left(\frac{h}{L_{n}}+\frac{x_{n}}{L_{n}}+\frac{x_{n-1}}{L_{n-1}}\right)\left(l_{k}-l_{n-i}\right):\right. \\
0 \leq k \leq n-m, 2 \leq i \leq m-1\}] .
\end{gathered}
$$

We continue this procedure and get for all $p, 0 \leq p \leq m-2$ : fixing the $x_{n-p}$-coordinate where

$$
-h \frac{L_{n-p}}{L_{n}}-L_{n-p}\left(\sum_{l=0}^{p-1} \frac{x_{n-l}}{L_{n-l}}\right) \leq x_{n-p} \leq 0
$$

in $S_{n-p}^{n-m}$ we obtain $P_{n-p-1}^{n-m}$ and after shifting we get $S_{n-p-1}^{n-m}$ with

$$
\begin{gathered}
S_{n-p-1}^{n-m}=\operatorname{co}\left[\left\{l_{k}\left(s-\sum_{l=0}^{p} \frac{x_{n-l}}{L_{n-l}}\right): 0 \leq k \leq n-m\right\}\right. \\
\cup\left\{l_{k}\left(s-\sum_{l=0}^{p} \frac{x_{n-l}}{L_{n-l}}\right)+\left(l_{k}-l_{n-i}\right)\left(\frac{h}{L_{n}}+\sum_{l=0}^{p} \frac{x_{n-l}}{L_{n-l}}\right):\right. \\
0 \leq k \leq n-m, p+1 \leq i \leq m-1\}] .
\end{gathered}
$$

Especially for $p=m-2$ we get the $(n-m+1)$-dimensional polytope

$$
S_{n-m+1}^{n-m}=c o\left[\left\{l_{k}\left(s-\sum_{l=0}^{m-2} \frac{x_{n-l}}{L_{n-l}}\right): 0 \leq k \leq n-m\right\}\right.
$$




$$
\begin{aligned}
& \cup\left\{l_{k}\left(s-\sum_{l=0}^{m-2} \frac{x_{n-l}}{L_{n-l}}\right)+\left(l_{k}-l_{n-m+1}\right)\left(\frac{h}{L_{n}}+\sum_{l=0}^{m-2} \frac{x_{n-l}}{L_{n-l}}\right):\right. \\
& 0 \leq k \leq n-m\}] .
\end{aligned}
$$

Observe that the points $l_{k}\left(s-\sum_{l=0}^{m-2}\left(x_{n-l} / L_{n-l}\right)\right), 0 \leq k \leq n-m$, span a regular $(n-m)$ dimensional simplex with side length $\left(s-\sum_{l=0}^{m-2}\left(x_{n-l} / L_{n-l}\right)\right)$ in $\mathbf{R}^{n-m+1}$ sitting in the hyperplane which has the $x_{n-m+1}$-coordinate equal to 0 . Likewise the points

$$
l_{k}\left(s-\sum_{l=0}^{m-2} \frac{x_{n-l}}{L_{n-l}}\right)+\left(l_{k}-l_{n-m+1}\right)\left(\frac{h}{L_{n}}+\sum_{l=0}^{m-2} \frac{x_{n-l}}{L_{n-l}}\right), \quad 0 \leq k \leq n-m,
$$

span a regular $(n-m)$-dimensional simplex in $\mathbf{R}^{n-m+1}$ sitting in the hyperplane which has the $x_{n-m+1}$-coordinate equal to $-\left(h / L_{n}+\sum_{l=0}^{m-2}\left(x_{n-l} / L_{n-l}\right)\right)$.

The sidelength of this simplex is

$$
\begin{gathered}
\| l_{k}\left(s-\sum_{l=0}^{m-2} \frac{x_{n-l}}{L_{n-l}}\right)+\left(l_{k}-l_{n-m+1}\right)\left(\frac{h}{L_{n}}+\sum_{l=0}^{m-2} \frac{x_{n-l}}{L_{n-l}}\right)-l_{k+1}\left(s-\sum_{l=0}^{m-2} \frac{x_{n-l}}{L_{n-l}}\right) \\
+\left(l_{k+1}-l_{n-m+1}\right)\left(\frac{h}{L_{n}}+\sum_{l=0}^{m-2} \frac{x_{n-l}}{L_{n-l}}\right) \|_{2} \\
=\left(s+\frac{h}{L_{n}}\right)\left\|l_{k}-l_{k+1}\right\|_{2}=s+\frac{h}{L_{n}} .
\end{gathered}
$$

Hence $S_{n-m+1}^{n-m}$ is the convex combination of two regular $(n-m)$-dimensional simplices sitting in parallel hyperplanes and, consequently,

$$
\begin{aligned}
\operatorname{vol}_{n-m+1}\left(S_{n-m+1}^{n-m}\right)= & \frac{\prod_{i=1}^{n-m+1} L_{i}}{(n-m+1) !}\left(\left(s+\frac{h}{L_{n}}\right)^{n-m+1}-\left(s+\sum_{l=0}^{m-2} \frac{-x_{n-l}}{L_{n-l}}\right)^{n-m+1}\right) \\
= & \frac{\prod_{i=1}^{n-m+1} L_{i}}{(n-m+1) !} \sum_{r=1}^{n-m+1}\left(\begin{array}{c}
n-m+1 \\
r
\end{array}\right)(-1)^{r+1} \\
& \times\left(\frac{h}{L_{n}}+\sum_{l=0}^{m-2} \frac{x_{n-l}}{L_{n-l}}\right)^{r}\left(s+\frac{h}{L_{n}}\right)^{n-m+1-r} .
\end{aligned}
$$

Now we integrate successively with respect to the $x_{n-m+2^{-}}, x_{n-m+3^{-}}, \ldots, x_{n}$-coordinates and get

$$
\begin{aligned}
\operatorname{vol}_{n}\left(P^{n-m}\right)= & \frac{\prod_{i=1}^{n} L_{i}}{(n-m+1) !} \sum_{r=1}^{n-m+1}\left(\begin{array}{c}
n-m+1 \\
r
\end{array}\right)(-1)^{r+1}\left(s+\frac{h}{L_{n}}\right)^{n-m+1-r} \\
& \times\left(\frac{h}{L_{n}}\right)^{r+m-1} \frac{1}{(r+1)(r+2) \cdots(r+m-1)}
\end{aligned}
$$


Recall that $h=n \delta / \operatorname{vol}_{n-1}\left(S_{n-1}\right)$ and collect terms in powers of $\delta$ to obtain

$$
\operatorname{vol}_{n}\left(P^{n-m}\right)=\sum_{k=m}^{n}\left(\begin{array}{l}
n \\
k
\end{array}\right) \frac{\delta^{k}}{\left(\operatorname{vol}_{n}\left(S_{n}\right)\right)^{k-1}} \sum_{i=0}^{k-m}(-1)^{i}\left(\begin{array}{c}
k \\
m+i
\end{array}\right) \text {. }
$$

Proof of Theorem 1. Taking into account that there are $\left(\begin{array}{c}n+1 \\ n-m+1\end{array}\right)$ faces of dimension $(n-m)$ of $S_{n}$, the proof of Theorem 1 follows immediately from Lemma 4 and

$$
\operatorname{vol}_{n}\left(S_{n}^{\delta}\right)-\operatorname{vol}\left(S_{n}\right)=\sum_{m=1}^{n}\left(\begin{array}{c}
n+1 \\
n-m+1
\end{array}\right) \sum_{k=m}^{n}\left(\begin{array}{l}
n \\
k
\end{array}\right) \frac{\delta^{k}}{\left(\operatorname{vol}_{n}\left(S_{n}\right)\right)^{k-1}} \sum_{i=0}^{k-m}(-1)^{i}\left(\begin{array}{c}
k \\
m+i
\end{array}\right) .
$$

We then collect terms in powers of $\delta$ and obtain the expression of the theorem.

\section{Acknowledgments}

I would like to thank R. Howard and E. Lutwak for drawing my attention to these problems. I would also like to thank the referee for many helpful suggestions.

\section{References}

[G] P. M. Gruber: Aspects of approximation of convex bodies, Handbook of Convex Geometry, vol. A, North-Holland, Amsterdam, 1993, pp. 321-345.

[Le] K. Leichtweiss: Zur Affinoberfläche konvexer Körper, Manuscripta Math. 56 (1986), 429-464.

[Lu] E. Lutwak: Extended affine surface area, Adv. in Math. 85 (1991), 39-68.

[S] C. Schütt: The convex floating body and polyhedral approximation, Israel J. Math. 73 (1991), 65-77.

[SW] C. Schütt, E. Werner: The convex floating body, Math. Scand. 66 (1990), 275-290.

[W] E. Werner: Illumination bodies and affine surface area, Studia Math. 110(3), (1994), 257-269.

Received August 29, 1994, and in nevised form February 23, 1995. 